ABHANDLUNGEN DER SÄCHSISCHEN AKADEMIE DER WISSENSCHAFTEN ZU LEIPZIG Mathematisch-naturwissenschaftliche Klasse Band $57 \cdot$ Heft 3

\title{
Umweltgestaltung in der Bergbaulandschaft
}

Herausgegeben von

CHRISTIAN HÄNSEL

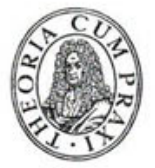




\title{
ABHANDLUNGEN DER SÄCHSISCHEN AKADEMIE \\ DER WISSENSCHAFTEN ZU LEIPZIG
}

\author{
Mathematisch-naturwissenschaftliche Klasse
}

\section{Band 51}

Heft 1 Beiträge zur Chemie und Technologie der Brennstoffe

Gedenkschrift für Prof. Dr. techn. habil. ANTON LISSNER

Herausgegeben von Prof. Dr.-Ing. Dr. Ing. eh. ERICH RAMMLER

1971. 146 Seiten - 1 Titelbild - 57 Textabbildungen, davon 6 auf 3 Tafeln -35 Tabellen $-4^{\circ}$

Heft 2 Dr. GŨNTER HERRMANN, Beiträge zur Bestimmung von Schwefeldioxid in der Atmosphäre und Herstellung von Schwefeldioxid-Luft-Gemischen

1971. 179 Seiten - 74 Abbildungen, davon 13 auf 10 Kunstdrucktafeln -41 Tabellen $-4^{\circ}$

Heft 3 Prof. Dr. med. GEORG MERREM, Die parasagittalen Meningiome

Zum Gedächtnis des Begründers der deutschen Neurochirurgie Prof. Dr. FEDOR KRAUSE 1857-1937

1971. 38 Seiten -20 Abbildungen -1 Tabelle $-4^{\circ}$

Heft 4 Prof. Dr. MAXIMILIAN KLINKOWSKI, Epidemien und Pandemien pflanzenpathogener Krankheitserreger in ihrer Beziehung zum Menschen

1971. 54 Seiten - 41 Abbildungen -4 Tabellen $-4^{\circ}$

Heft 5 Prof. Dr. Gustav E. R. Schulze und Dr. Peter Paufler, Die plastische Verformung "spröder" intermetallischer Verbindungen und ihre Elementarprozesse

1972. 24 Seiten - 14 Abbildungen - 11 Tabellen $-4^{\circ}$

Band 52

Heft 1 Prof. Dr. KuRT MoTHEs, Über sekundäre Pflanzenstoffe 1972. 29 Seiten -18 Abbildungen $-4^{\circ}$

Heft 2 Prof. Dr. Dietrich UhLMANN, Störungen des biologischen Gleichgewichts in Gewässern 1973. 17 Seiten - 16 Abbildungen $-4^{\circ}$

Heft 3 Felix Lampadius, Beitrag zum Nachweis der Wertminderung des Waldes als Folge von Immissionseinwirkung

1974. 27 Seiten - 4 Abbildungen - 8 Tabellen $-4^{\circ}$

Heft 4 Dr. MANFRED KLOSE, Beiträge zur D- und V-Struktur von Wasser und speziellen wäßrigen

Elektrolytlösungen

1974. 63 Seiten -22 Abbildungen -21 Tabellen $-4^{\circ}$

Heft 5 Prof. Dr. med. HORST HANSON, Eiweißumsatz und Differenzierungsvorgänge tierischer Zellen

1974. 26 Seiten - 13 Abbildungen - 9 Tabellen $-4^{\circ}$

\section{Band 58}

Heft 1 3. Internationales Symposium über Probleme der Passivität von Metallen (Technische Universität Dresden, 29.-31. 5. 1975)

1979. 171 Seiten - 150 Abbildungen, davon 26 auf 10 Tafeln -27 Tabellen $-4^{\circ}$ 
ABHANDLUNGEN DER SÄCHSISCHEN AKADEMIE DER WISSENSCHAFTEN ZU LEIPZIG

Mathematisch-naturwissenschaftliche Klasse

Band $57 \cdot$ Heft 3

\title{
Umweltgestaltung in der Bergbaulandschaft
}

\author{
Herausgegeben von CHRISTIAN HÄNSEL
}

Mit 26 Abbildungen und 7 Tabellen 
Ergebnisse einer Tagung der Kommission für spezielle Umweltprobleme der Sächsischen Akademie der Wissenschaften zu Leipzig vom 5.-8. Juli 1989 in Großsteinberg bei Leipzig

Manuskript vorgelegt in der Sitzung am 9. Februar 1990

Manuskript eingereicht am 24. April 1990

Druckfertig erklärt am 2.8.1991

Herausgeber:

Prof. Dr. Christian Hänsel, Leipzig

Das vorliegende Werk wurde sorgfältig erarbeitet. Dennoch übernehmen Autoren, Herausgeber und Verlag für die Richtigkeit von Angaben, Hinweisen und Ratschlägen sowie für eventuelle Druckfehler keine Haftung.

Lektorat: Dipl.-Met. Heide Deutscher

Die Deutsche Bibliothek - CIP-Einheitsaufnahme

Umweltgestaltung in der Bergbaulandschaft : mit 7 Tabellen ;

[Ergebnisse einer Tagung der Kommission für Spezielle

Umweltprobleme der Sächsischen Akademie der

Wissenschaften zu Leipzig vom 5. - 8. Juli 1989 in

Grosssteinberg bei Leipzig]. - Berlin : Akad.-Verl., 1991

(Abhandlungen der Sächsischen Akademie der Wissenschaften zu

Leipzig, Mathematisch-Naturwissenschaftliche Klasse ; Bd. 57, H. 3)

ISBN 3-05-501291-7

NE: Hänsel, Christian [Hrsg.]; Sächsische Akademie der Wissenschaften

(Leipzig) / Kommission für Spezielle Umweltprobleme; Sächsische

Akademie der Wissenschaften 〈Leipzig〉/ Mathematisch-

Naturwissenschaftliche Klasse: Abhandlungen der Sächsischen ...

ISBN 3-05-501291-7

ISSN 0365-6470

(C) Akademie Verlag GmbH, Berlin 1991

Erschienen in der Akademie Verlag GmbH, O-1086 Berlin (Federal Republic of Germany), Leipziger Str. 3-4

Gedruckt auf säurefreiem Papier

Alle Rechte, insbesondere die der Übersetzung in andere Sprachen, vorbehalten. Kein Teil dieses Buches darf ohne schriftliche Genehmigung des Verlages in irgendeiner Form - durch Photokopie, Mikroverfilmung oder irgendein anderes Verfahren - reproduziert oder in eine von Maschinen, insbesondere von Datenverarbeitungsmaschinen, verwendbare Sprache übertragen oder übersetzt werden. Die Wiedergabe von Warenbezeichnungen, Handelsnamen oder sonstigen Kennzeichen in diesem Buch berechtigt nicht zu der Annahme, daß diese von jedermann frei benutzt werden dürfen. Vielmehr kann es sich auch dann um eingetragene Warenzeichen oder sonstige gesetzlich geschützte Kennzeichen handeln, wenn sie nicht eigens als solche markiert sind.

Gesamtherstellung: Druckhaus Köthen $\mathrm{GmbH}$

Printed in the Federal Republic of Germany 